\title{
Visceral and somatic hypersensitivity, autonomic cardiovascular dysfunction and low-grade inflammation in a subset of irritable bowel syndrome patients*
}

\author{
Liang LIU ${ }^{1,2}$, Bei-ni LIU ${ }^{1,2}$, Shuo CHEN ${ }^{1,2}$, Miao WANG ${ }^{1}$, Yang LIU ${ }^{1,3}$, Yan-li ZHANG ${ }^{\dagger 11}$, Shu-kun YAO $\$ 1,2$ \\ ( ${ }^{1}$ Department of Gastroenterology, China-Japan Friendship Hospital, Beijing 100029, China) \\ ( ${ }^{2}$ Graduate School, Peking Union Medical College \& Chinese Academy of Medical Sciences, Beijing 100730, China) \\ ( ${ }^{3}$ Beijing University of Chinese Medicine, Beijing 100029, China) \\ †E-mail: janetzhyl@gmail.com; ysk12329@126.com
}

Received May 21, 2014; Revision accepted Aug. 30, 2014; Crosschecked Sept. 20, 2014

\begin{abstract}
The pathophysiology of irritable bowel syndrome (IBS) is complex and not fully understood, so the aim of this study was to evaluate whether visceral and somatic hypersensitivity, autonomic cardiovascular dysfunction, and low-grade inflammation of the gut wall are associated with diarrhea-predominant IBS (D-IBS). Sixty-two patients with D-IBS and 20 control subjects participated in the study. Using the ascending method of limits (AML) protocol, we demonstrated that D-IBS patients had significantly lower sensory thresholds compared with healthy controls $(P<0.001)$. Using diverse methods, especially the ischemic sensitivity test, for the first time in China, we confirmed that D-IBS patients have somatic hypersensitivity. They had a significantly higher systolic blood pressure and heart rate after a cold stimulus, indicative of autonomic cardiovascular dysfunction. Compared with the control group, D-IBS patients had a significantly higher level of calprotectin $(P<0.001)$. We also found significant correlations between visceral and somatic hypersensitivity, visceral hypersensitivity and autonomic cardiovascular dysfunction, and somatic hypersensitivity and autonomic cardiovascular dysfunction. Our findings may provide valuable suggestions for the treatment of D-IBS.
\end{abstract}

Key words: Irritable bowel syndrome (IBS), Visceral hypersensitivity, Somatic hypersensitivity, Autonomic cardiovascular dysfunction, Low-grade inflammation doi: 10.1631 jzus.B1400143

Document code: A

CLC number: R574.4

\section{Introduction}

Irritable bowel syndrome (IBS) is one of the most common functional bowel disorders seen by gastroenterologists and is characterized by intermittent episodes of abdominal pain or/and distension, without any organic diseases (Liu et al., 2014). IBS is

\footnotetext{
${ }^{\ddagger}$ Corresponding authors

* Project supported by the National Science \& Technology Pillar Program during the Twelfth Five-year Plan Period of China (No. 2014BAI08B02) and the National Science \& Technology Major Projects (Major Drug Discovery) of China (No. 2012ZX09103201-021) (C) Zhejiang University and Springer-Verlag Berlin Heidelberg 2014
}

common in Western countries, where the incidence is up to $20 \%$. In recent years, the morbidity in Asian countries has been rising year by year, and has now nearly reached that of Western countries (Zhong and Hou, 2012; Song et al., 2012; Omagari et al., 2013). Without biological markers, the diagnosis of IBS depends mainly on the patient's symptoms. According to Rome III criteria, IBS patients are subdivided into diarrhea-predominant IBS (D-IBS), constipationpredominant IBS (C-IBS), mixed type IBS (M-IBS), and un-subtyped IBS (U-IBS). The pathophysiology of IBS is complex and not fully understood; a centralperipheral regulatory mechanism may be involved (Zhou et al., 2010). 
Although the pathophysiology of IBS is unclear, visceral hypersensitivity is one of the accepted mechanisms, and may have some relationships with the severity of the symptoms (Zhou et al., 2009). However, previous evaluations of visceral sensitivity have typically been based on "rapid phasic distension", and Harraf et al. (1998) claimed that visceral hypersensitivity could not be induced by the "ascending method of limits (AML)" protocol. On the other hand, compared with normal controls, some IBS patients exhibit a high proportion of somatic hypersensitivity symptoms, such as migraine headaches, heartburn, back pain, and muscle pain. Most previous investigations of somatic hypersensitivity involved only a single stimulus method, and the conclusions were contradictory (Whitehead et al., 1990; Zighelboim et al., 1995). Whether autonomic cardiovascular dysfunction is involved in the pathological process of IBS is controversial (Elsenbruch and Orr, 2001; Tousignant-Laflamme et al., 2006; Cheng et al., 2013). Finally, a growing body of research evidence suggests that damage to the intestinal mucosal barrier and low-grade inflammation of the gut wall are related to the pathogenesis of IBS (Peuhkuri et al., 2010; Snoek et al., 2010; Liu et al., 2012).

The aims of this study were: (1) to evaluate visceral sensitivity in IBS patients using the AML method and to explore patterns of stimulus in the evaluation of somatic sensitivity; (2) to determine whether IBS patients have autonomic cardiovascular dysfunction; (3) to verify if IBS patients have intestinal mucosal barrier damage and low-grade inflammation of the gut wall; and, (4) to identify relationships among visceral hypersensitivity, somatic hypersensitivity, autonomic cardiovascular dysfunction, intestinal mucosal barrier damage, and low-grade inflammation of the gut wall in IBS patients, and to determine if the related experimental parameters are correlated with the severity of symptoms.

\section{Materials and methods}

\subsection{Participants}

This study was carried out from September 2013 to May 2014 in the Gastroenterology Kinetic Laboratory of the China-Japan Friendship Hospital, Beijing, China. Sixty-two patients with D-IBS and 20 control subjects participated in the study. IBS patients were recruited at the endoscopy center or the outpatient clinic, and control subjects were via advertisements. The participants gave their written informed consent prior to data collection. The study was approved by the Ethics Committee of the China-Japan Friendship Hospital, China. The diagnosis of all IBS patients was made by the same doctor and was based on the Rome III criteria, with normal colonoscopy examination. None of the controls had organic gastrointestinal disorder, abnormal laboratory findings, weight loss, anemia, bloody stools, family history of cancer, or other alarming signs, and those who were pregnant or had previously undergone gastrointestinal surgery were excluded from the study. None of the participants had taken serotonin antagonists, tricyclic antidepressants, pain medications, proton pump inhibitors, or serotonin uptake inhibitors for at least three weeks prior to the study.

All sessions were conducted between 8:00 am and 6:00 pm to avoid circadian rhythm effects. Demographics, such as the gender, age, height, weight, resting heart rate, and blood pressure, of all subjects were recorded. A research coordinator provided detailed information to the subjects about how to collect stool and urine specimens (for the purpose of gut wall inflammation and intestinal mucosal barrier function tests). Specimens were temporarily stored in a fridge at $-80{ }^{\circ} \mathrm{C}$ for enzyme-linked immunosorbent assay (ELISA) testing. Fig. 1 shows the experimental process.

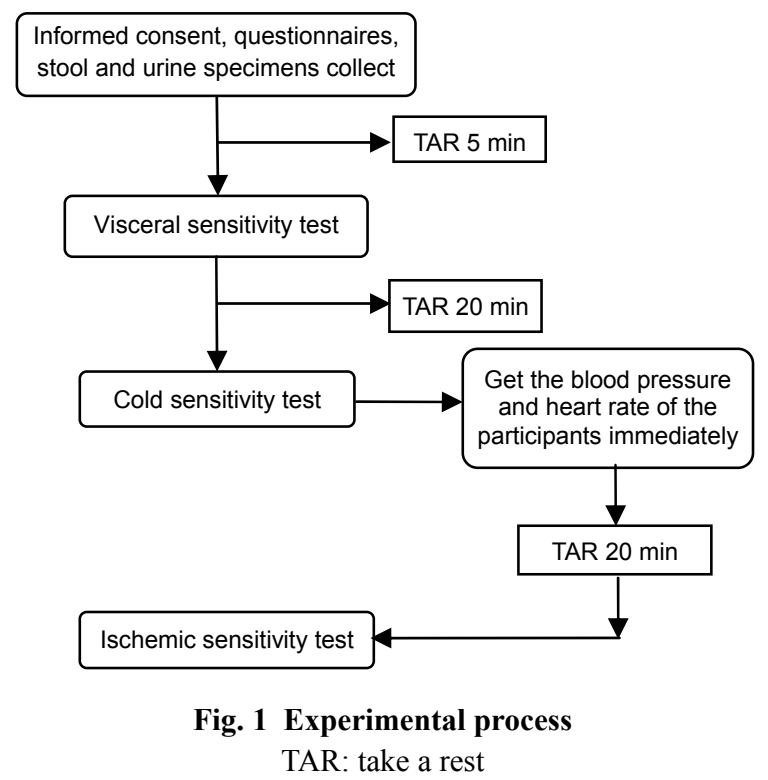




\subsection{Measures}

\subsubsection{Hospital anxiety and depression scale (HADS)}

HADS is commonly used for estimating emotional disorders, and can be divided into anxiety (HAD-A) and depression (HAD-D) subscales (Dong et al., 2013). For each subscale, there are seven questions. A total score of $0-7$ is classed as normal, $8-10$ as borderline, and $>11$ as severe.

\subsection{2 "Severity of IBS" questionnaire}

The IBS severity questionnaire, which includes five items, was used to assess the severity of symptoms. Each item has a 100-point scale and the final score is the sum of scores from the five items. Patients were defined as having mild IBS with the score of 75-175, moderately severe IBS with the score 175-300, or severe IBS of the score $>300$ (Francis et al., 1997; Singh et al., 2012).

\subsubsection{Visceral sensitivity test (AML protocol)}

A barostat (G\&J Electronics Inc., Willowdale, Ontario, Canada) was used for this test. All subjects were placed in the left lateral decubitus position in a hospital bed. A lubricated polyethylene balloon (with a maximum volume of $600 \mathrm{ml}$ ) secured to a rectal catheter was inserted into the rectum such that the distal attachment site was $4 \mathrm{~cm}$ from the anal verge. The barostat was used to inflate the balloon. Distensions of 30-s duration were followed by $30-\mathrm{s}$ rest intervals, starting at the operating pressure and progressively increasing in 1-mmHg steps. In the process of the test, patients were asked to report on sensory thresholds: initial feeling (IF), initial defecate feeling (IDF), embarrassment of defecation (EOD), and maximum of tolerance (MOT). If the MOT or the maximum pressure $(50 \mathrm{mmHg})$ was reached, the balloon was deflated automatically. All subjects were examined by the same physician.

2.2.4 Cold sensitivity test and autonomic cardiovascular reaction

A cold pain test is commonly used to evaluate somatic sensitivity (Whitehead et al., 1990; Zighelboim et al., 1995). In this study, all subjects were asked to immerse their feet in water at $4{ }^{\circ} \mathrm{C}$ (the volume of cold water was such that the temperature change was not obvious in a short time). Subjects were instructed to keep their feet in the water for up to 5 min or until the pain became intolerable. The cold pain threshold (CPTh) was recorded as the time when the subjects first felt pain and the cold pain tolerance (CPTo) was recorded as the time when they removed their feet from the cold water. An automatic blood pressure monitor (Andon Health Co., Ltd., Tianjin, China) was then used immediately to record the systolic blood pressure (SBP) and heart rate (HR) of the participants.

\subsubsection{Ischemic sensitivity test}

A sphygmomanometer (Liankang Co., Ltd., Shanghai, China) was used for the ischemic sensitivity test. The right arm of the patient was elevated above heart level for $1 \mathrm{~min}$, after which the blood flow was occluded with a blood pressure cuff. The cuff was inflated to $240 \mathrm{mmHg}$ and kept at that level. Participants were told to perform 20 hand grip exercises (50\% of maximum grip strength) with 2 -s duration and 4-s rest interval. The ischemic pain threshold (IPTh) was recorded as the time when they first felt pain, and their ischemic pain tolerance (IPTo) was recorded when the pain was intolerable or when $15 \mathrm{~min}$ had elapsed (Zhou et al., 2010).

2.2.6 Gut wall low-grade inflammation and intestinal mucosal barrier function

The ELISA test (double antibody sandwich method) was used to quantify the level of calprotectin in stool specimens, and the levels of intestinal fatty acid binding protein (I-FABP), intestinal bile acid binding protein (I-BABP), and liver fatty acid binding protein (L-FABP) in urine specimens. Calprotectin results are expressed as $\mathrm{mg} / \mathrm{kg}$, and urine specimen results are expressed as $\mathrm{ng} / \mathrm{ml}$. The ELISA procedures were followed according to the recommendations of the manufacturer (Beijing Xinfangcheng Biotechnology Co., Ltd., China).

\subsection{Statistical analysis}

Statistical analysis was performed using SPSS Version 19.0 (IBM, USA). Distributions of demographic characteristics were analyzed by Pearson's $\chi^{2}$ or Fisher's exact test. Student's $t$-test was used to compare group differences between continuous variables. Data are presented as mean \pm standard deviation (SD). Pearson's correlation coefficients among 
visceral hypersensitivity, somatic hypersensitivity, autonomic cardiovascular dysfunction, mucous membrane barrier damage, and low-grade inflammation of the gut wall were determined. Spearman's correlations were used to determine the relationships between experimental parameters and the severity of symptoms for IBS patients. $P<0.05$ was considered statistically significant.

\section{Results}

\subsection{Demographic characteristics of participants}

All participants completed the study. The demographic characteristics of participants are listed in Table 1. The gender distribution, height, weight, HAD-A, and HAD-D were similar between the D-IBS and control groups $(P>0.05)$. D-IBS patients were much older on average than the healthy controls. Of the 62 D-IBS patients, 42 had moderately severe IBS, 20 had severe IBS, and none had mild IBS.

\subsection{Visceral sensitivity test}

In the D-IBS group, the four kinds of sensory thresholds (IF, IDF, EOD, and MOT) were significantly lower than in healthy controls $(P<0.001$; Table 2). There was no gender difference among the four kinds of sensory thresholds in the D-IBS group.

\subsection{Somatic sensitivity test}

With the cold sensitivity test, D-IBS patients took a shorter time to reach both the CPTh and CPTo $(P<0.001$; Table 3$)$. Likewise, the time to reach both the IPTh and IPTo was much shorter in the D-IBS group $(P<0.001$; Table 3$)$. No gender difference was found in somatic sensitivity among D-IBS patients.

\subsection{Autonomic cardiovascular reaction}

There were no significant differences in the SBP or HR in the resting state between the two groups $(P>0.05$; Table 4). However, the SBP and HR after the cold sensitivity test were much higher in D-IBS patients $(P<0.001$; Table 4$)$.

\subsection{Low-grade inflammation of the gut wall and intestinal mucosal barrier function tests}

Stool calprotectin was higher in D-IBS patients. Compared with the healthy controls, the D-IBS

Table 1 Demographic characteristics of participants

\begin{tabular}{ccccccc}
\hline Group & Male/female & Height $(\mathrm{m})$ & Weight $(\mathrm{kg})$ & Age $($ year $)$ & HAD-A & HAD-D \\
\hline D-IBS $(n=62)$ & $35 / 27$ & $1.68 \pm 0.08$ & $66.5 \pm 14.9$ & $38.4 \pm 10.1$ & $3.53 \pm 3.30$ & $3.74 \pm 3.55$ \\
Control $(n=20)$ & $12 / 8$ & $1.69 \pm 0.07$ & $62.8 \pm 10.4$ & $27.9 \pm 3.4$ & $3.25 \pm 2.47$ & $2.20 \pm 2.09$ \\
\hline$P$-value & 0.826 & 0.442 & 0.306 & $<0.001$ & 0.726 & 0.070 \\
\hline
\end{tabular}

HAD-A: the hospital anxiety and depression scale-anxiety; HAD-D: the hospital anxiety and depression scale-depression. Data are presented as mean $\pm \mathrm{SD}$

Table 2 Results of visceral sensitivity tests

\begin{tabular}{ccccc}
\hline Group & IF $(\mathrm{mmHg})$ & IDF $(\mathrm{mmHg})$ & EOD $(\mathrm{mmHg})$ & MOT $(\mathrm{mmHg})$ \\
\hline D-IBS $(n=62)$ & $5.21 \pm 2.20$ & $8.97 \pm 2.81$ & $12.63 \pm 3.66$ & $16.75 \pm 4.59$ \\
Control $(n=20)$ & $8.97 \pm 1.93$ & $13.11 \pm 1.97$ & $17.93 \pm 2.45$ & $23.04 \pm 3.38$ \\
\hline$P$-value & $<0.001$ & $<0.001$ & $<0.001$ & $<0.001$ \\
\hline
\end{tabular}

IF: initial feeling; IDF: initial defecate feeling; EOD: embarrassment of defecation; MOT: maximum of tolerance. Data are presented as mean \pm SD

Table 3 Results of somatic sensitivity tests

\begin{tabular}{ccccc}
\hline Group & CPTh $(\mathrm{s})$ & CPTo $(\mathrm{s})$ & \multicolumn{1}{c}{ IPTh $(\mathrm{s})$} & IPTo $(\mathrm{s})$ \\
\hline D-IBS $(n=62)$ & $21.97 \pm 9.75$ & $61.00 \pm 42.78$ & $77.21 \pm 40.85$ & $188.36 \pm 73.74$ \\
Control $(n=20)$ & $44.79 \pm 14.35$ & $128.93 \pm 49.73$ & $171.79 \pm 46.48$ & $414.39 \pm 79.09$ \\
\hline$P$-value & $<0.001$ & $<0.001$ & $<0.001$ & $<0.001$ \\
\hline
\end{tabular}

CPTh: cold pain threshold; CPTo: cold pain tolerance; IPTh: ischemic pain threshold; IPTo: ischemic pain tolerance. Data are presented as mean \pm SD 
patients also showed elevated levels of I-FABP, I-BABP, and L-FABP, but these differences were not statistically significant $(P>0.05$; Table 5$)$.

\subsection{Pearson's and Spearman's correlations for IBS patients}

For D-IBS patients, significant correlations were found between visceral and somatic hypersensitivity, and visceral hypersensitivity and autonomic cardiovascular dysfunction $(P<0.001$; Table 6). Similarly, somatic hypersensitivity and autonomic cardiovascular dysfunction had a significant negative correlation $(P<0.001$; Table 7). Finally, except for weak significant correlations with HAD-A $(r=0.225$; $P<0.001)$, HAD-D $(r=0.302 ; P<0.001)$, and CPTh $(r=-0.345 ; P<0.001)$, we found no significant correlations between the severity of symptoms and experimental parameters.

\section{Discussion}

Unlike most previous studies, in this study, we used the AML method and demonstrated that compared with healthy controls, D-IBS patients had significantly lower sensory thresholds, i.e., they had visceral hypersensitivity. Harraf et al. (1998) claimed that visceral hypersensitivity could not be induced by the AML protocol. Compared with "rapid phasic distension", which mainly stimulates the intestinal serosa receptors, the main effect of AML is through the stimulation of the intestinal mucosa and submucosa receptors. These receptors are involved in different nerve conduction pathways while also participating in intestinal perception (Mertz et al., 1995; Dorn et al., 2007). Visceral hypersensitivity is a biological marker in some IBS cases, especially D-IBS patients. In contrast to Posserud et al. (2007), we found no gender difference in the visceral hypersensitivity. Gender distribution was balanced in our IBS group, while Posserud et al. (2007)'s group was composed of almost all women. This may have caused a gender bias, and so comparisons with our study may be inappropriate.

Many IBS patients exhibit a variety of somatic symptoms, such as migraine headaches, heartburn, back pain, and muscle pain. In this study, we confirmed that D-IBS patients have somatic hypersensitivity using both cold and ischemic sensitivity tests. Our results were similar to those of previous studies (Zhou et al., 2009; 2010). To our knowledge, our work is the first study to use diverse methods, especially the ischemic sensitivity test, to examine somatic sensitivity in China. Unlike the cold sensitivity test which activates superficial $\mathrm{A} \delta$ fibers in the skin, the ischemic sensitivity test stimulates a large number of thick C-fibers in muscle and produces a deep and diffuse pain (Zuo et al., 2006; Zhou et al., 2010). In view of the above features, the ischemic sensitivity test may be more accurate for the evaluation of somatic sensitivity.

Table 4 Autonomic cardiovascular reaction after cold sensitivity test

\begin{tabular}{cccccc}
\hline Group & \multicolumn{2}{c}{ Resting state } & & \multicolumn{2}{c}{ After cold sensitivity test } \\
\cline { 1 - 2 } \cline { 5 - 6 } \cline { 5 - 6 } & $\mathrm{SBP}(\mathrm{mmHg})$ & HR $($ time $/ \mathrm{min})$ & & $\mathrm{SBP}(\mathrm{mmHg})$ & HR (time $/ \mathrm{min})$ \\
\hline D-IBS $(n=62)$ & $115.96 \pm 10.95$ & $72.08 \pm 4.96$ & & $138.40 \pm 11.47$ & $92.03 \pm 8.60$ \\
Control $(n=20)$ & $112.93 \pm 4.96$ & $73.00 \pm 5.75$ & & $119.04 \pm 6.09$ & $77.50 \pm 6.84$ \\
\hline$P$-value & 0.160 & 0.411 & & $<0.001$ & $<0.001$
\end{tabular}

SBP: systolic blood pressure; HR: heart rate. Data are presented as mean \pm SD

Table 5 Results of stool and urine specimens

\begin{tabular}{ccccc}
\hline Group & Calprotection $(\mathrm{mg} / \mathrm{kg})$ & I-FABP $(\mathrm{ng} / \mathrm{ml})$ & I-BABP $(\mathrm{ng} / \mathrm{ml})$ & L-FABP $(\mathrm{ng} / \mathrm{ml})$ \\
\hline D-IBS $(n=62)$ & $55.70 \pm 10.13$ & $1475.10 \pm 281.02$ & $1011.87 \pm 223.20$ & $762.45 \pm 174.08$ \\
Control $(n=20)$ & $45.72 \pm 5.67$ & $1425.62 \pm 300.66$ & $955.99 \pm 180.00$ & $705.30 \pm 166.44$ \\
\hline$P$-value & $<0.001$ & 0.503 & 0.312 & 0.201 \\
\hline
\end{tabular}

I-FABP: intestinal fatty acid binding protein; I-BABP: intestinal bile acid binding protein; L-FABP: liver fatty acid binding protein. Data are presented as mean $\pm \mathrm{SD}$ 
Table 6 Pearson's correlation coefficients for D-IBS patients between visceral hypersensitivity and somatic hypersensitivity, and visceral hypersensitivity and autonomic cardiovascular dysfunction

\begin{tabular}{lclll}
\hline \multirow{2}{*}{ Parameter } & \multicolumn{4}{c}{ Pearson's correlation coefficient } \\
\cline { 2 - 5 } & IF & IDF & EOD & MOT \\
\hline IPTh & 0.519 & 0.497 & 0.523 & 0.537 \\
IPTo & 0.582 & 0.577 & 0.591 & 0.602 \\
CPTh & 0.658 & 0.549 & 0.518 & 0.512 \\
HR after cold & -0.523 & -0.566 & -0.559 & -0.629 \\
$\quad$ sensitivity test & & & & \\
\hline
\end{tabular}

IF: initial feeling; IDF: initial defecate feeling; EOD: embarrassment of defecation; MOT: maximum of tolerance; IPTh: ischemic pain threshold; IPTo: ischemic pain tolerance; CPTh: cold pain threshold; HR: heart rate. Data are presented as mean \pm SD. All $P<0.001$

Table 7 Pearson's correlation coefficients for D-IBS patients between somatic hypersensitivity and autonomic cardiovascular dysfunction

\begin{tabular}{ccc}
\hline \multirow{2}{*}{ Parameter } & \multicolumn{2}{c}{ Pearson's correlation coefficient } \\
\cline { 2 - 3 } & SBP after cold & HR after cold \\
& sensitivity test & sensitivity test \\
\hline IPTh & -0.642 & -0.683 \\
IPTo & -0.661 & -0.727 \\
\hline
\end{tabular}

IPTh: ischemic pain threshold; IPTo: ischemic pain tolerance; SBP: systolic blood pressure; HR: heart rate. All $P<0.001$

In our study, we found that compared with the control group, D-IBS patients had a significantly higher SBP and HR after cold stimulus, indicating the presence of autonomic cardiovascular dysfunction in the IBS group. Few studies have found autonomic nervous system (ANS) dysfunction in response to a painful somatic stimulus, but our results suggest that when exposed to an external stimulus, such as cold water, the regulation of the ANS was in an abnormal state in IBS patients. This phenomenon suggests that the dysfunction of central-peripheral nerve regulation mechanisms may be involved in the pathogenesis of IBS.

A number of IBS, especially D-IBS, patients have a history of acute gastroenteritis (Zhong and Hou, 2012; Liu et al., 2012). Inflammation, even low-grade, can send many systemic and local signals, which may cause changes in the intestine (such as the dysfunction of digestion and absorption). Calprotectin, which is a protein released by white blood cells and stable in faeces, can indicate inflammation wherever it occurs in the digestive tract (Derikx et al.,
2010). In this study, compared with the healthy group, D-IBS patients had a significantly higher level of calprotectin. Some studies have found that faeces calprotectin was not higher in IBS patients. The recruitment of only D-IBS patients and the relatively large sample size in our study may explain this discrepancy. Three isoforms of FABP (I-FABP, I-BABP, and L-FABP) are present in the intestinal villus (Derikx et al., 2010). The urinary concentration of FABP is a useful marker for evaluating intestinal mucosal barrier function. In our results, the concentrations of the three isoforms of FABP were all higher in D-IBS patients than those in the control group, but the differences were not statistically significant. We speculate that if the sample size had been larger, a significant difference may have been found.

To our surprise, in this study, we showed significant correlations between visceral and somatic hypersensitivity, visceral hypersensitivity and autonomic cardiovascular dysfunction, and somatic hypersensitivity and autonomic cardiovascular dysfunction, which have not been found before to our knowledge. Our results also suggest recommendations for the treatment of IBS. Previously, too much attention has been given to only intestinal symptoms. At present, the conventional drugs used in the treatment of IBS include: laxatives, antidiarrheals, prokinetics, probiotics, and antispasmodics. The role of these drugs is mainly to relieve the patient's gastrointestinal symptoms, but the curative effects have been disappointing (Rahimi and Abdollahi, 2012). The possible reason is that many patients with IBS also exhibit a wide variety of extra-intestinal symptoms. From our results, individualized treatments (such as cognitive behavioral therapy, hypnotherapy, dietotherapy, relaxation, and stress management in improving the function of the ANS, and traditional Chinese medicine and acupuncture to relieve somatic hypersensitivity) are recommended to alleviate the extra-intestinal symptoms (Rahimi and Abdollahi, 2012; Wilkins et al., 2012; Chang, 2014). According to our data, the remission of extra-intestinal symptoms may be associated with the alleviation of gastrointestinal symptoms in D-IBS patients.

Unlike previous studies, no strong correlations between the severity of symptoms and experimental parameters were found in our study (Zhou et al., 2009), indicating that the mechanisms involved in the 
expression of symptoms are still complicated and inadequately understood.

A strength of our study was that our analysis excluded the confounding effects of mental factors. However, some limitations should be considered. First, the survey was restricted to D-IBS, so the findings may not be generally applicable to other subtypes. Second, based on the current research, we could not explain the results in terms of molecular mechanisms, a future area of research focus. Third, there were age differences between the IBS and control groups. Sometimes, this is unavoidable in reallife clinical research, but no significant effects of gender were found in our two groups. Furthermore, age difference is not an influential factor in the evaluation of experimental parameters such as visceral hypersensitivity (Posserud et al., 2007).

In summary, our results showed that patients with D-IBS showed evidences of visceral hypersensitivity, somatic hypersensitivity, autonomic cardiovascular dysfunction, and low-grade inflammation in the digestive tract. We also found significant correlations between visceral and somatic hypersensitivity, visceral hypersensitivity and autonomic cardiovascular dysfunction, and somatic hypersensitivity and autonomic cardiovascular dysfunction, which may provide valuable suggestions for the treatment of D-IBS.

\section{Compliance with ethics guidelines}

Liang LIU, Bei-ni LIU, Shuo CHEN, Miao WANG, Yang LIU, Yan-li ZHANG, and Shu-kun YAO declare that they have no conflict of interest.

All procedures followed were in accordance with the ethical standards of the responsible committee on human experimentation (institutional and national) and with the Helsinki Declaration of 1975, as revised in 2008 (5). Informed consent was obtained from all patients for being included in the study.

\section{References}

Chang, F.Y., 2014. Irritable bowel syndrome: the evolution of multi-dimensional looking and multidisciplinary treatments. World J. Gastroenterol., 20(10):2499-2514. [doi: 10.3748/wjg.v20.i10.2499]

Cheng, P., Shih, W., Alberto, M., et al., 2013. Autonomic response to a visceral stressor is dysregulated in irritable bowel syndrome and correlates with duration of disease. Neurogastroenterol. Motil., 25(10):650-659. [doi:10. 1111/nmo.12177]

Derikx, J.P., Luyer, M.D., Heineman, E., et al., 2010.
Non-invasive markers of gut wall integrity in health and disease. World J. Gastroenterol., 16(42):5272-5279. [doi: 10.3748/wjg.v16.i42.5272]

Dong, Y.Y., Chen, F.X., Yu, Y.B., et al., 2013. A school-based study with Rome III criteria on the prevalence of functional gastrointestinal disorders in Chinese college and university students. PLOS ONE, 8(1):e54183. [doi:10. 1371/journal.pone.0054183]

Dorn, S.D., Palsson, O.S., Thiwan, S.I., et al., 2007. Increased colonic pain sensitivity in irritable bowel syndrome is the result of an increased tendency to report pain rather than increased neurosensory sensitivity. Gut, 56(9):1202-1209. [doi:10.1136/gut.2006.117390]

Elsenbruch, S., Orr, W.C., 2001. Diarrhea- and constipationpredominant IBS patients differ in postprandial autonomic and cortisol responses. Am. J. Gastroenterol., 96(2):460-466. [doi:10.1111/j.1572-0241.2001.03526.x]

Francis, C.Y., Morris, J., Whorwell, P.J., 1997. The irritable bowel severity scoring system: a simple method of monitoring irritable bowel syndrome and its progress. Aliment. Pharmacol. Ther., 11(2):395-402. [doi:10.1046/j.13652036.1997.142318000.x]

Harraf, F., Schmulson, M., Saba, L., et al., 1998. Subtypes of constipation predominant irritable bowel syndrome based on rectal perception. Gut, 43(3):388-394. [doi:10.1136/ gut.43.3.388]

Liu, L., Cai, X., Yan, J., et al., 2012. In vivo and in vitro antinociceptive effect of Fagopyrum cymosum (Trev.) Meisn extracts: a possible action by recovering intestinal barrier dysfunction. Evid. Based Complement. Alternat. Med., 2012:983801. [doi:10.1155/2012/983801]

Liu, L., Xiao, Q.F., Zhang, Y.L., et al., 2014. A cross-sectional study of irritable bowel syndrome in nurses in China: prevalence and associated psychological and lifestyle factors. J. Zhejiang Univ.-Sci. B (Biomed. \& Biotechnol.), 15(6):590-597. [doi:10.1631/jzus.B1300159]

Mertz, H., Naliboff, B., Munakata, J., et al., 1995. Altered rectal perception is a biological marker of patients with irritable bowel syndrome. Gastroenterology, 109(1): 40-52. [doi:10.1016/0016-5085(95)90267-8]

Omagari, K., Murayama, T., Tanaka, Y., et al., 2013. Mental, physical, dietary, and nutritional effects on irritable bowel syndrome in young Japanese women. Intern. Med., 52(12): 1295-1301. [doi:10.2169/internalmedicine.52.0248]

Peuhkuri, K., Vapaatalo, H., Korpela, R., 2010. Even lowgrade inflammation impacts on small intestinal function. World J. Gastroenterol., 16(9):1057-1062. [doi:10.3748/ wjg.v16.i9.1057]

Posserud, I., Syrous, A., Lindstrom, L., et al., 2007. Altered rectal perception in irritable bowel syndrome is associated with symptom severity. Gastroenterology, 133(4): 1113-1123. [doi:10.1053/j.gastro.2007.07.024]

Rahimi, R., Abdollahi, M., 2012. Herbal medicines for the management of irritable bowel syndrome: a comprehensive review. World J. Gastroenterol., 18(7):589-600. [doi:10.3748/wjg.v18.i7.589] 
Singh, P., Agnihotri, A., Pathak, M.K., et al., 2012. Psychiatric, somatic and other functional gastrointestinal disorders in patients with irritable bowel syndrome at a tertiary care center. J. Neurogastroenterol. Motil., 18(3):324-331. [doi:10.5056/jnm.2012.18.3.324]

Snoek, S.A., Verstege, M.I., Boeckxstaens, G.E., et al., 2010. The enteric nervous system as a regulator of intestinal epithelial barrier function in health and disease. Expert Rev. Gastroenterol. Hepatol., 4(5):637-651. [doi:10.1586/ egh.10.51]

Song, S.W., Park, S.J., Kim, S.H., et al., 2012. Relationship between irritable bowel syndrome, worry and stress in adolescent girls. J. Korean Med. Sci., 27(11):1398-1404. [doi:10.3346/jkms.2012.27.11.1398]

Tousignant-Laflamme, Y., Goffaux, P., Bourgault, P., et al., 2006. Different autonomic responses to experimental pain in IBS patients and healthy controls. J. Clin. Gastroenterol., 40(9):814-820. [doi:10.1097/01.mcg.0000225607. 56352.ce]

Whitehead, W.E., Holtkotter, B., Enck, P., et al., 1990. Tolerance for rectosigmoid distention in irritable bowel syndrome. Gastroenterology, 98(5):1187-1192.
Wilkins, T., Pepitone, C., Alex, B., et al., 2012. Diagnosis and management of IBS in adults. Am. Fam. Phys., 86(5): 419-426.

Zhong, L., Hou, X., 2012. Pathophysiologic findings of irritable bowel syndrome in China. J. Neurogastroenterol. Motil., 18(1):19-33. [doi:10.5056/jnm.2012.18.1.19]

Zhou, Q., Zhang, B., Verne, G.N., 2009. Intestinal membrane permeability and hypersensitivity in the irritable bowel syndrome. Pain, 146(1-2):41-46. [doi:10.1016/j.pain. 009.06.017]

Zhou, Q., Fillinqim, R.B., Riley, J.L., et al., 2010. Central and peripheral hypersensitivity in the irritable bowel syndrome. Pain, 148(3):454-461. [doi:10.1016/j.pain.2009. 2.005]

Zighelboim, J., Talley, N.J., Phillips, S.F., et al., 1995. Visceral perception in irritable bowel syndrome. Rectal and gastric responses to distension and serotonin type 3 antagonism. Dig. Dis. Sci., 40(4):819-827. [doi:10.1007/BF02064986]

Zuo, X.L., Li, Y.Q., Shi, L., et al., 2006. Visceral hypersensitivity following cold water intake in subjects with irritable bowel syndrome. J. Gastroenterol., 41(4):311-317. [doi:10.1007/s00535-005-1766-x]

\section{中文楥要:}

本文题目: 肠易激综合征亚型患者具有肠道和躯体高敏感, 自主心血管功能紊乱以及肠道低浓度炎症 Visceral and somatic hypersensitivity, autonomic cardiovascular dysfunction and low-grade inflammation in a subset of irritable bowel syndrome patients

研究目的: 明确腹泻型肠易激综合征患者是否具有肠道和躯体高敏感、自主心血管功能紊乱以及肠道 低浓度炎症。

创新要点: 发现腹泻型肠易激综合征患者具有自主心血管功能紊乱和肠道低浓度炎症（这两方面在之 前研究中尚存争议）。此外，发现腹泻型肠易激综合征患者的肠道高敏感和躯体高敏感、 肠道高敏感和自主心血管功能紊乱、躯体高敏感和自主心血管紊乱之间存在相关性, 为患 者的治疗提供参考。

研究方法: 2013 年 9 月至 2014 年 5 月, 62 名腹泻型肠易激综合征患者和 20 名健康志愿者参与到了本 研究。所有受试者接受并顺利完成了相关检查和问卷填写。利用恒压器（渐至极限法）检 查我们发现腹泻型肠易激综合征患者具有肠道高敏感。同时, 采用冷水刺激法和缺血刺激 法探明腹泻型肠易激综合征患者具有躯体高敏感。

重要结论: 腹泻型肠易激综合征患者具有肠道和躯体高敏感、自主心血管功能紊乱以及肠道低浓度炎 症。肠道高敏感和身体高敏感、肠道高敏感和自主心血管功能紊乱、躯体高敏感和自主心 血管紊乱之间存在相关性。

关键词组: 肠易激综合征（IBS）；肠道高敏感；躯体高敏感；自主心血管功能紊乱；低浓度炎症 